\title{
High granularity scintillating fiber trackers based on Silicon Photomultiplier
}

\section{A. Papa}

Paul Scherrer Institut, Villigen, Switzerland E-mail: angela.papa@psi.ch

\section{E. Ripiccini*}

Istituto Nazionale di Fisica Nucleare Sez. di Roma, Rome, Italy E-mail:

emanuele.ripiccinieromal.infn.it

\section{G. Rutar}

Paul Scherrer Institut, Villigen and Swiss Federal Institute of Technology ETH, Zurich,

Switzerland E-mail: giada.rutarepsi.ch

Scintillating fibers coupled to photosensors provide flexible, fast and high granularity detectors which are able to work in high rate environment. We will report about the performances obtained with multi-layer detector prototype based on $250 \mu \mathrm{m}$ multi-clad square scintillating fibers, 20 $\mathrm{cm}$ long, coupled to $1.3 \times 1.3 \mathrm{~mm}^{2}$ active area silicon photomultiplier (SiPMs). Current measurements show results never reached up to now: high detection efficiency for minimum ionizing particles (m.i.p.) already for a single layer (>90\%, mean collected light/fiber 8.5 phe). Also a good spatial resolution can be achieved by keeping the optical cross-talk between fibers at a negligible level $(<1 \%)$, a level has been achieved by coating the fibers with aluminum.. Finally, the time resolution of the order of $500 \mathrm{ps}$ has been achieved for m.i.p. in a single layer configuration. The resolution improves with increasing the number of detector layers. All measurements have been supported with a Monte Carlo simulation based on Geant 4 and a custom code, describing the response of the SiPMs.

International Conference on New Photo-detectors, PhotoDet2015

6-9 July 2015

Moscow, Troitsk,Russia

\footnotetext{
* Speaker.
} 


\section{Introduction}

The aim of this project is the realization of a tracker made of adjacent layers of the thinnest scintillating fibers available on the market $(250 \mu \mathrm{m})$ read-out by $1.3 \times 1.3 \mathrm{~mm}^{2}$ active area SiPMs (Hamamatsu S13360-1350CS) [1]. The requirements for this device are: a high flexibility, a fast read-out (it must be able to support a high particle rate $1 \mathrm{MHz} /$ fiber $)$, minimum thickness $(<0.003$ $X_{0}$ ), high detection efficiency ( $>90 \%$ ), high position resolution ( $50 \mu \mathrm{m}$ ) and high time resolution $(<500 \mathrm{ps})$.We developed and tested double and single read-out prototypes in different configurations, single fiber, array and telescope and also multi-layer prototype is almost ready for being tested. The single fiber prototype has been used to determine the mechanical solution which provided the most precise fiber SiPM coupling and, as a consequence, the highest detection efficiency $(>90 \%)$. The spatial resolution of a single layer is expected to be about $70 \mu \mathrm{m}$ which is the sigma of a uniform distribution with a width of $250 \mu \mathrm{m}$. Thanks to the multiple layers, this number can be reduced significantly, keeping the crosstalk at a negligible level and thanks to a high fabrication precision. The measurements performed with the array prototypes allowed us to understand how to minimize the optical crosstalk between the adjacent fibers and also how to realize an array with several fibers keeping the same performances for each fiber. The telescope prototype consisting of 3 arrays of fiber has been developed to study the multi staggered-layer performances in terms of detection efficiency, position and timing resolution. With prototype we could also achieve a high time resolution by exploiting the multi-hit information. We tested all the prototypes both in laboratory, using minimum ionizing electrons from the tail of the Sr90 spectrum, and during beam time, using low momentum (28 MeV/c, degraded for muons) positron and muon beam. Here we present the results obtained with the last telescope prototype. As the signal produced by a minimum ionizing positron (electron) is very low ( $<10$ photo-electrons) and it is also at the level of the SiPM dark current signal, the main challenge of this device is the detection of these particles with the highest reachable detection efficiency. This work can be applied for developments of a beam monitoring tool in MEG II experiment [2] and positron time detector in Mu3e experiment [3].

\section{Experimental setup}

The experimental setup consists of seven fibers arranged to obtain a telescope structure, see figure 1. Such configuration allowed us to study the section efficiency of the layer by setting a proper trigger that will be discussed in the next section. All the fibers are coated with Aluminum deposited using the vaporization technique. Both ends of each fiber are viewed by SiPMs, consequently each fiber is a single detector. We used Saint Gobain BCF-12 multi-clad square fibers [6] coupled to $1.3 \times 1.3 \mathrm{~mm}^{2}$ active area SiPMs from Hamamatsu [7]. The signal is amplified with a custom front-end electronics [4] based on mini circuit MAR-6 pre-amplifier and digitized with DRS 4 chip (5 Gs/s) developed at Paul Scherrer Institut [5].

Each end of the fiber is glued in the groove of the plexiglass frame and then polished by using a diamond tip. A PCB, where the SiPMs are soldered, is screwed to the side of the frame, see figure 2. Currently the precision of the relative alignment fiber-SiPM is of the order of $300 \mu \mathrm{m}$. In case of a weak signal, like the one produced by a minimum ionizing particle (MIP) crossing a 250 $\mu \mathrm{m}$ thick scintillating fiber, the detection efficiency is affected by the number of missed photons. 

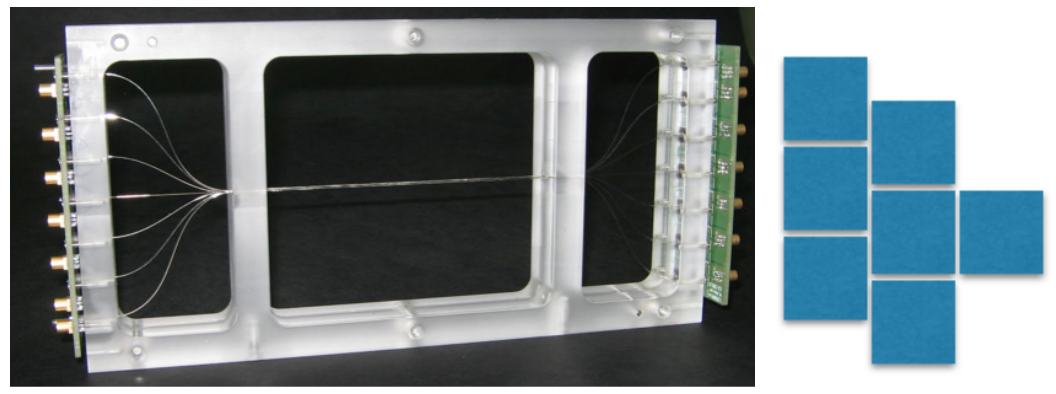

Figure 1: On the left a frontal view of the experimental setup. On the right, a schematic view of the fiber arrangement

All the SiPMs we used have a $550 \mu \mathrm{m}$ thick epoxy window over the active area. The photons are emitted by the fiber in a cone so that they illuminate a spot on the SiPM active surface with a size larger than $250 \mu \mathrm{m}$. Using $1 \times 1 \mathrm{~mm}^{2}$ active area SiPMs, we observed that a small misalignment between the fiber and the SiPM leads to a loss of light up to the $30 \%$ with respect to the ideal case. A Monte Carlo simulation shows how a $300 \mu \mathrm{m}$ misalignment between the fiber and the SiPM originates a light spread which is not fully contained on the SiPM active area. We found a good solution to this problem by using $1.3 \times 1.3 \mathrm{~mm}^{2}$ active area SiPMs, see figure 3 .
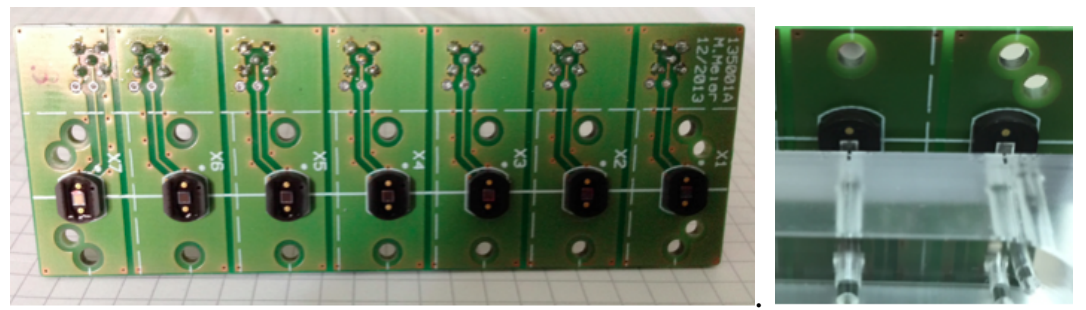

Figure 2: On the left, the SiPMs soldered on the PCB. On the right, detail of the fiber driven to the SiPM active area through the groove
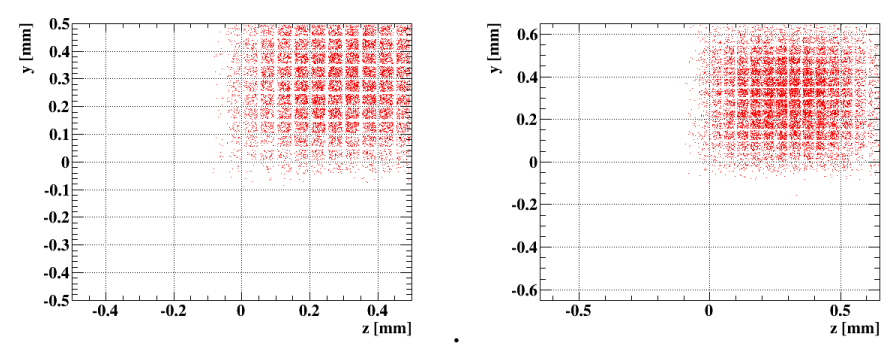

Figure 3: Simulation of the spatial distribution of the photon on the SiPM active area when a fiber-SiPM coupling misalignment of $300 \mu \mathrm{m}$ is applied on both axis. On the left the configuration with the $1 \times 1 \mathrm{~mm}^{2}$ active area SiPMs and on the right the configuration with the $1.3 \times 1.3 \mathrm{~mm}^{2}$ active area SiPMs 


\section{Detector performances}

\subsection{Light collection and detection efficiency}

For the measurement of the light collected in each fiber we used different sources: electrons from $90 \mathrm{Sr}$ (selecting the tail of the spectrum in order to have minimum ionizing particles), muons and positrons with a momentum of $28 \mathrm{MeV} / \mathrm{c}$. It is important to notice that positrons with this energy are near the minimum ionizing point. The average energy deposited in a $250 \mu \mathrm{m}$ thick fiber produces a weak signal which such that the SiPM dark current contamination is not negligible. An external trigger, which fixes the integration window of the waveform, reduces the probability of a dark current signal to occur down to less than $1 \%$. A typical trigger we used selects only particles passing trough the frontal single fiber of the telescope. An example of the spectrum obtained by summing the charge collected by the two SiPMs at the end of one fiber irradiated with $90 \mathrm{Sr}$ electrons is reported in figure 4. We have two spectra: the OR spectrum, where the charge of the two SiPMs are summed in logical OR, requiring that at least one SiPM fired and the AND spectra, where the charge of the two SiPMs are summed in logical AND, both SiPM fired. In figure 5 the charge spectrum obtained irradiating a single fiber with positrons and muons is reported. 28 $\mathrm{MeV} / \mathrm{c}$ momentum muons are ionizing particles. The average number of photoelectrons is 8.5 for the positrons and 85 for the muons, which is far away from the dark current contamination. We repeated the measurement for each fiber obtaining the same average number of photo electrons. In all these measurements the particles interact in the middle of the fiber. The detection efficiency
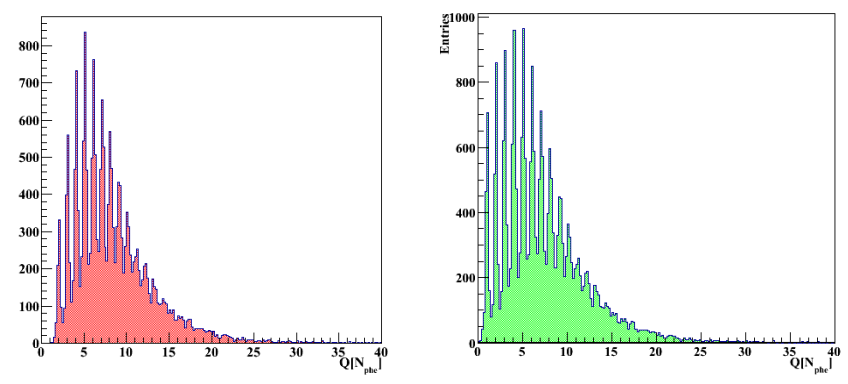

Figure 4: Calibrated charge spectra where the charges collected by the two SiPMs are summed using logical AND, on the left, and a logical OR, on the right.

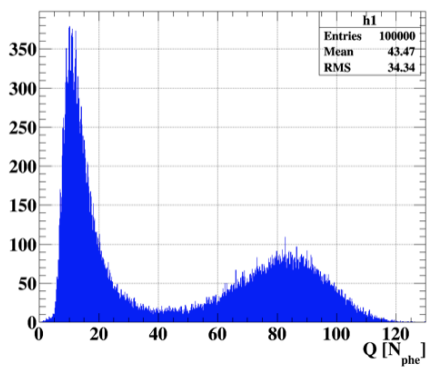

Figure 5: Calibrated charge spectrum of both muon and positron passing through the fiber.

was measured using both electrons/positrons and muons. The trigger system is made by the AND 
between the two SiPMs, with a high threshold ( 3 photoelectrons), of the fiber 1, which is the first element of the telescope irradiated by the beam. Such a trigger provides a geometrical constrain for the beam, in fact all the selected particles pass through the central fiber of the second layer and at least in one of the two fiber of the third layer. We measured the following detection efficiency values for MIPs:

- $\varepsilon^{A N D}=75 \%$ and $\varepsilon^{O R}=92 \%$ for the single fiber.

- $\varepsilon^{A N D}=79 \%$ and $\varepsilon^{O R}=97 \%$ for the array with three fibers.

For the single fiber the detection efficiency is defined as the ratio between the number of events with a charge higher than 0.5 photoelectrons and the number of total triggers, while for the array it is ratio between the sum of the number of events with a charge higher than 0.5 photoelectrons of each fiber of the layer and the number of total triggers as well. This detection efficiency is the highest ever measured with a $250 \mu \mathrm{m}$ thick fiber irradiated by a minimum ionizing particles. The optical crosstalk between adjacent fibers has been minimized by coating all the fibers with a layer of Aluminum deposited with the evaporation technique. Figure 6 shows the collected charge from a fired fiber with respect to the charge leaked to the adjacent fiber. The level of optical crosstalk was measured to be less than $1 \%$.

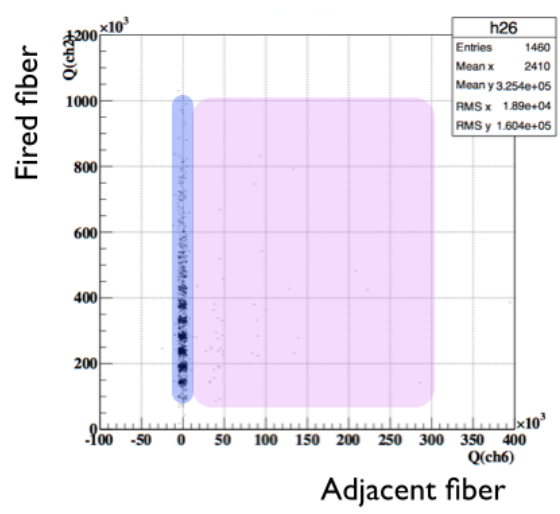

Figure 6: Collected charge from a fiber with respect to the charge collected from the adjacent one.

\subsection{Time measurements}

Another requirement for this device is the high time resolution. The measurement was done using $28 \mathrm{MeV} / \mathrm{c}$ positrons crossing two consecutive layers. Using a software constant fraction (usually $20 \%$ of the maximum peak) discriminator we can extract, event by event, the time of the waveforms of the two SiPMs instrumenting the ends of the fiber, $t_{a}$ and $t_{b}$. We calculate the average time of the particle crossing the fiber as $T=\frac{t_{a}+t_{b}}{2}$. The particle crosses two consecutive fibers respectively at $T_{1}$ and $T_{2}$. By fitting the distribution of the variable $\frac{T_{1}-T_{2}}{2}$ we measure the time resolution of the single fiber. We obtained $\sigma_{T}=580 \pm 5 \mathrm{ps}$, (see figure 7) irradiating the prototype both with electrons from $\mathrm{Sr} 90$ and positrons. 


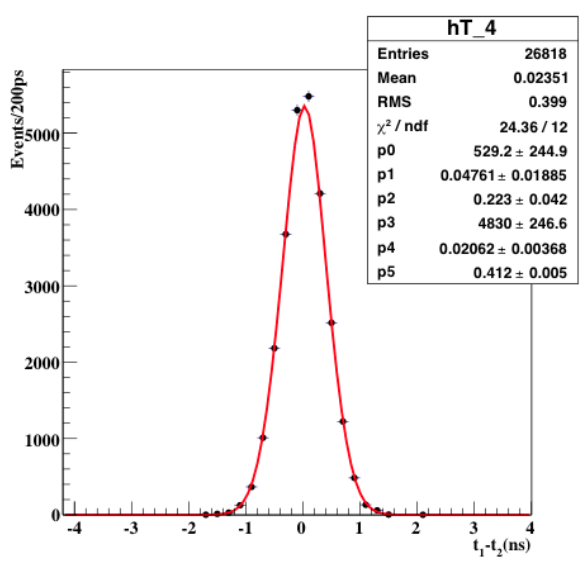

Figure 7: Distribution of times between two coincident fibers $\frac{T_{1}-T_{2}}{2}$.

\subsection{Beam monitoring}

Using a single fiber we measured the beam profile and rate both for muons and positrons, see figure 8 . The beam profile was measured for a few minutes while the pre existing procedure with beam devices requires more than a day to obtain the same results.
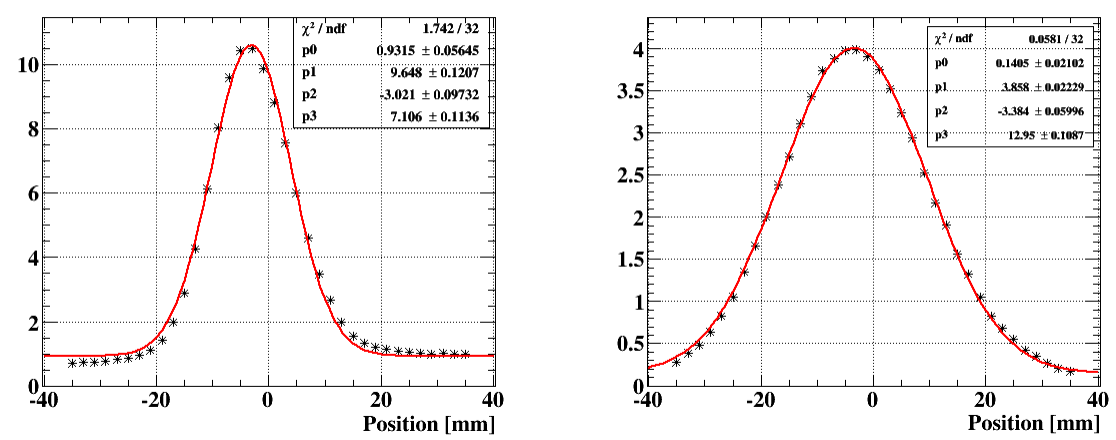

Figure 8: Positron beam profile on the vertical axis (left) and muon beam profile (right).

\section{Monte Carlo Simulation}

We also implemented a Monte Carlo simulation reproducing a $250 \mu \mathrm{m}$ thick fiber coupled to a $1.3 \times 1.3 \mathrm{~mm}^{2}$ active area SiPM. The software is divided in two parts. In the first part the geometry and the propagation of the scintillation photons through the fiber from the origin to the SiPM active area are simulated using Geant 4 . In the second part the time and space distribution of the photons on the SiPM active area is used by a program to reproduce the waveform according to [8]. Both the expected distributions of the number of photons at the end of the fiber and the simulated spectrum were obtained for $90 \mathrm{Sr}$ electrons crossing the fiber. In figure 9 on the left we can observe the attenuation of the light signal at different stages, starting from the number of photons at the end of the fiber to number of fired pixels which produce the signal. The red and the black histograms 
are quite perfectly superimposed, it means that the saturation effect is negligible. In figure 9 on the right the simulated spectra, obtained assuming a crosstalk probability of $1 \%$, is compared to the measured one. The comparison shows a clear discrepancy in the range populated by the first and the second photoelectron which is recovered attenuating the light of $20 \%$ in the first part of the code.
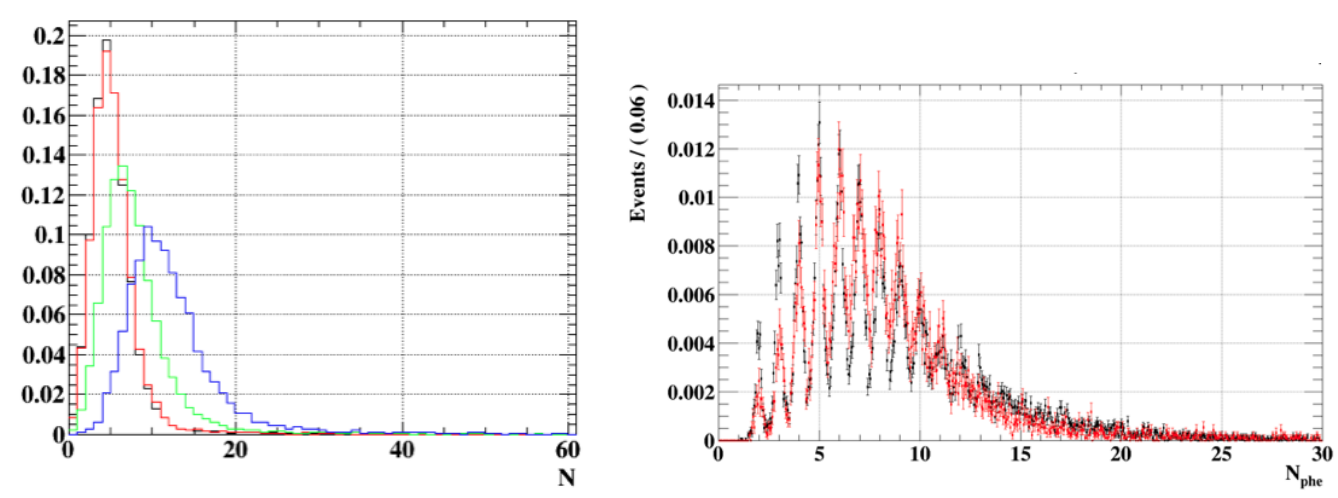

Figure 9: On the left the attenuation of the signal at different stages from the fiber to the SiPM pixel in the single read-out configuration: distribution of the number of photons at the end of the fiber (blue histogram), distribution of the number of photons on the SiPM active area (green histogram), distribution of the number of photons which produced an avalanche (red histogram), distribution of the number of fired pixels (black histogram). On the right the comparison between charge spectrum from Sr90 data, black line, and simulated one, red line.

\section{Conclusion}

We have proven the scintillating fibers single layer detector as the effective device to monitor beam under rates up to $100 \mathrm{MHz}$. We studied a multi-layer configuration as a stand alone timing detector as possible a complement tracker either for scintillating fiber trackers or as a complement trackers based on different technology. The following performances have been achieved:

- Light collected in average: 8.5 photoelectrons with minimum ionizing electrons/positrons and 85 photoelectrons with $28 \mathrm{MeV} / \mathrm{c}$ muons.

- High detection efficiency: $\varepsilon($ AND) $79 \% \varepsilon(\mathrm{OR}) 97 \%$.

- Good time resolution: 580 ns for single fiber.

- Ability to measure beam profile in short time.

- Expected space resolution with 3 layers is better than $70 \mu \mathrm{m}$.

\section{References}

[1] A. Papa et al., A multi-purposed detector with silicon photomultiplier readout of scintillating fibers,Nucl. Instrum. Meth. A 787 (2015) 130. 
[2] A. M. Baldini et al., MEG Upgrade Proposal, arXiv:1301.7225 [physics.ins-det].

[3] A. Blondel et al.,Research Proposal for an Experiment to Search for the Decay $\mu \rightarrow e e e$, arXiv:1301.6113 [physics.ins-det].

[4] A. Stoykov et al., NIMA 695 (2012) 202

[5] S. Ritt, NIMA (2002) 520

[6] http://www.detectors.saint-gobain.com/fibers.aspx

[7] https://www.hamamatsu.com/eu/en/product/category/3100/4004/4113/index.html

[8] J. Pulko et al., Monte-Carlo model of a SiPM coupled to a scintillating crystal Instrumentation, JINST 7 (2012) P02009. 